\title{
Broken time-reversal symmetry in Josephson junction involving two-band superconductors
}

\author{
T.K. $\mathrm{Ng}^{1}$, and N. Nagaosa ${ }^{2,3}$ \\ ${ }^{1}$ Department of Physics, Hong-Kong University of Science and Technology, \\ Kawloon, Hong Kong Peoples $R$ China \\ 2 Department of Applied Physics, The University of Tokyo, \\ 7-3-1 Hongo, Bunkyo-ku, Tokyo 113-8656, Japan \\ ${ }^{3}$ Cross Correlated Materials Research Group (CMRG), \\ ASI, RIKEN, WAKO 351-0198, Japan
}

\begin{abstract}
A novel time-reversal symmetry breaking state is found theoretically in the Josephson junction between the two-gap superconductor and the conventional s-wave superconductor. This occurs due to the frustration between the three order parameters analogous to the two antiferromagnetically coupled XY-spins put under a magnetic field. This leads to the interface states with the energies inside the superconducting gap. Possible experimental observations of this state with broken timereversal symmetry are discussed.
\end{abstract}


The complex behaviors of the superconductors beyond the BCS theory are now a focus of condensed matter physics. Among them, the superconductivity characterized by more than one order parameters, i.e., the multi-gap superconductors, is an intriguing and hot topic. Historically, the inter-band mechanism of the pairing for such a case has been proposed long ago $\frac{1}{2}$. A representative example of the multi-gap superconductor is $\mathrm{MgB}_{2} \frac{2}{2}$ where the specific heat $\underline{\underline{3}}$, the tunneling $\underline{\underline{4}}$, and angle-resolved photoemission spectroscopy (ARPES) $)^{\underline{5}}$ have revealed the different gap energies for $\sigma$ - and $\pi$-bands. The magnitudes of the gaps were analyzed by the first-principles band structure calculation $\underline{6}$ and are found to be strongly momentum- and band- dependent. This two-gap behavior is attributed to the strong coupling of the $\sigma$-band to the bond stretching phonon mode $\mathrm{e}^{\underline{6}}$.

The multi-gap structure should be common in the superconductors with the orbital degeneracy and/or the many electron/hole pockets, which is the case for the newly found high temperature superconductor iron pnictides ${ }^{7}$. In these compounds, there are two small electron pockets around M-points and two hole pockets around $\Gamma$-point ${ }^{\underline{8}}$. There are many proposals for the gap pairing symmetry ${ }^{\underline{9}}$, and one possibility is that a full gap opens for each pocket, which is consistent with the recent ARPES in $\mathrm{Ba}_{0.6} \mathrm{~K}_{0.4} \mathrm{Fe}_{2} \frac{10}{}$, although ARPES cannot determine the relative sign of the order parameters. Therefore, the determination of the relative sign of the order parameters on the pockets is now an important issue to fix the microscopic mechanism for the superconductivity. A clue has been given by the resonant magnetic scattering $\underline{\underline{11}}$ which is attributed to the triplet exciton in the superconducting state. A comparison with the earlier theoretical analysis $\frac{12}{2}$ suggests that the relative sign of the order parameter is minus in $\mathrm{Ba}_{0.6} \mathrm{~K}_{0.4} \mathrm{Fe}_{2}$.

In this paper, we explore theoretically a novel phenomenon in the two-gap superconductors when coupled to another single-band superconductor by the Josephson effect. The two bands are assumed to have separated Fermi surfaces in $\vec{k}$-space and are coupled only through electron-electron interaction. We shall assume for simplicity that the superconducting order parameters on all Fermi surfaces have $s$-symmetry, although most of our results can be generalized to order-parameters with other symmetries as well. We start with the phenomenological Ginzburg-Landau (GL) free energy density of the two band superconductor.

$F_{0}\left(\psi_{1}, \psi_{2}\right)=\alpha_{1}(T)\left|\psi_{1}\right|^{2}+K_{1}\left|\vec{D} \psi_{1}\right|^{2}+\beta_{1}\left|\psi_{1}\right|^{4}+\alpha_{2}(T)\left|\psi_{2}\right|^{2}+K_{2}\left|\vec{D} \psi_{2}\right|^{2}+\beta_{2}\left|\psi_{2}\right|^{4}-J\left[\psi_{1}^{*} \psi_{2}+\psi_{2}^{*} \psi_{1}\right]$ 
where $\psi_{1(2)}$ is the superconducting order parameter for band $1(2)$ and $\vec{D}=-i[\nabla-2 e i \vec{A} / \hbar c]$. In general the two superconducting bands are coupled by an internal Josephson-coupling term $\sim J$ as a result of electron-electron interaction. Writing $\psi_{1(2)}=\left|\psi_{1(2)}\right| e^{i \theta_{1(2)}}$ and minimizing the energy with respect to $\theta_{1(2)}$ in the absence of magnetic field it is easy to see that $\psi_{1}$ and $\psi_{2}$ are of the same $\operatorname{sign}\left(\theta_{1}=\theta_{2}(\bmod 2 \pi)\right)$ if $J>0$, and are of opposite sign $\left(\theta_{1}=\theta_{2}+\pi(\bmod 2 \pi)\right)$ if $J<0$. The question is whether there exists any non-trivial physical consequences associated with this relative sign, in particular when $J<0$ and $\psi_{1} \sim-\psi_{2}$ ?

In the following we shall show that the spontaneous time-reversal symmetry breaking occurs at the Josephson junction between the two-band superconductor and another singleband $s$-wave superconductor when the sign of $J$ is negative. To be concrete we assume that the single-band superconductors is located at the left side $(x<0)$ of the Josephson junction, and the two-band superconductor is located on the right $(x>0)$. The two superconductors are weakly coupled by Josephson effect, and the total free energy density of the system is $F=F_{0} \theta(x)+F_{s} \theta(-x)+F_{J}$, where

$$
F_{s}\left(\psi_{s}\right)=\alpha_{s}(T)\left|\psi_{s}\right|^{2}+K_{s}\left|\vec{D} \psi_{s}\right|^{2}+\beta_{s}\left|\psi_{s}\right|^{4}
$$

is the usual Ginsburg-Landau free energy for the single-band $s$-wave superconductor, and

$$
F_{J}=-\left(T_{1}\left[\psi_{1}^{*} \psi_{s}+\psi_{s}^{*} \psi_{1}\right]+T_{2}\left[\psi_{2}^{*} \psi_{s}+\psi_{s}^{*} \psi_{2}\right]\right) \delta(x)
$$

is the Josephson coupling between the two superconductors. $T_{1(2)}$ represents the coupling of the single-band superconductor to the two separate bands. We note that $T_{1}$ and $T_{2}$ are both positive according to the perturbation theory in the tunnelling matrix elements between the two superconductors. The relative sign between $\psi_{1}$ and $\psi_{2}$ is "unknown" to the single-band superconductor in the Josephson effect.

The non-trivial effect associated with the Josephson junction can be seen by minimizing the free energy of the system with respect to the phases of the superconductors, assuming that the amplitudes of the order parameters are constants. In the absence of magnetic field the GL free energy density with phase variables only is

$$
\begin{aligned}
F \sim & \bar{F}+\theta(x)\left(-2 \tilde{J} \cos \left(\theta_{1}-\theta_{2}\right)+\tilde{K}_{1}\left(\nabla \theta_{1}\right)^{2}+\tilde{K}_{2}\left(\nabla \theta_{2}\right)^{2}\right) \\
& -2 \delta(x)\left(\tilde{T}_{1} \cos \left(\theta_{1}-\theta_{s}\right)+\tilde{T}_{2} \cos \left(\theta_{2}-\theta_{s}\right)\right)+\tilde{K}_{s}\left(\nabla \theta_{s}\right)^{2}
\end{aligned}
$$

where $\bar{F}$ is the part of free energy density which is independent of $\theta$ 's. $\tilde{\theta}_{1(2)}$ is defined at $x \geq 0$ and $\theta_{s}$ is defined as $x \leq 0 . \tilde{J}=J\left|\psi_{1}\right|\left|\psi_{2}\right|, \tilde{T}_{1(2)}=T_{1(2)}\left|\psi_{s}\right|\left|\psi_{1(2)}\right|$ and $\tilde{K}_{\nu}=K_{\nu}\left|\psi_{\nu}\right|^{2}$, 


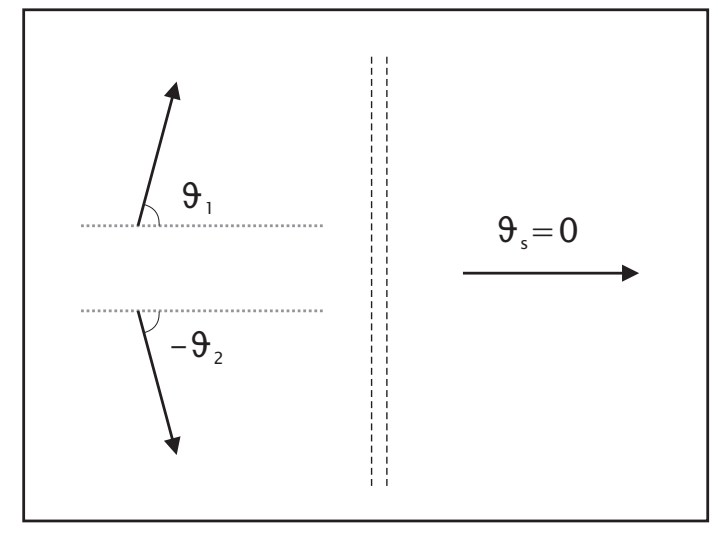

FIG. 1: A schematic representation of canted state in the effective classical spin model for the Josephson junction between a two band superconductor and another single-band superconductor. The coupling between the two spins $\left(\theta_{1}, \theta_{2}\right)$ are antiferromagnetic $(J<0)$ but they are both coupling ferromagnetically to a magnetic field along $\hat{x}$-direction $\left(\theta_{s}=0\right)$

$\nu=1,2, s$. We shall take $\theta_{s}(x=0)=0$ in the following. This is allowed because the overall phase of the system is a pure gauge. First we note that for $J>0$ the phase configuration which minimizes the above free energy is simply $\theta_{1}=\theta_{2}=\theta_{s}=0$ and there is no nontrivial effect associated with the Josephson junction. The situation becomes different for $J<0$ where the phases are "frustrated" because of sign difference between $J$ and $T_{1(2)}$. To see what could happen we note that the system is similar to a system of two classical spins $A$ and $B$ that are antiferromagnetically coupled and are put under a weak magnetic field in $\hat{x}$ direction representing the Josephson coupling of the system to the single-band superconductor. If the coupling of spin $A$ to magnetic field is much stronger than that of spin $B\left(T_{1}>>T_{2}\right)$, spin $A$ will be allied to the magnetic field with spin $B$ remaining antiparallel to spin $A$ in the ground state, i.e. $\theta_{1}=0, \theta_{2}=\pi$ in the corresponding Josephson junction problem. The converse $\left(\theta_{2}=0, \theta_{1}=\pi\right)$ is true if $T_{2}>>T_{1}$. This is the first possible state. Notice however that if the couplings of the two spins to the magnetic field are similar, there will be no preferred spin to the magnetic field and a second type of state where the two spins take angles $\theta_{1(2)} \sim \pm \pi / 2 \mp \delta \theta$ will be formed (see Fig.1).

The free energy (44) cannot be minimized exactly to obtain the two types of phase structures. We shall treat the free energy approximately in the following by writing $\theta_{1}=\theta_{0}+\tilde{\theta}_{1}$, $\theta_{2}=\theta_{0}-\pi+\tilde{\theta}_{2}$ and expand the free energy to order $\tilde{\theta}_{1(2)}^{2}$. The approximation can be justified in the limit of weak-Josephson coupling as we shall see later. 
Minimizing the resulting approximate free energy $F\left(\theta_{0}, \tilde{\theta}\right)$ with respect to $\tilde{\theta}$ 's we obtain

$$
\begin{aligned}
\tilde{J}\left(\tilde{\theta}_{1}-\tilde{\theta}_{2}\right)+\tilde{K}_{1} \nabla^{2} \tilde{\theta}_{1} & =\tilde{T}_{1} \delta(x)\left(\sin \theta_{0}+\cos \theta_{0} \tilde{\theta}_{1}\right) \\
\tilde{J}\left(\tilde{\theta}_{2}-\tilde{\theta}_{1}\right)+\tilde{K}_{2} \nabla^{2} \tilde{\theta}_{2} & =-\tilde{T}_{2} \delta(x)\left(\sin \theta_{0}+\cos \theta_{0} \tilde{\theta}_{2}\right), \\
\tilde{K}_{s} \nabla^{2} \theta_{s} & =-\delta(x)\left(\tilde{T}_{1}\left(\sin \theta_{0}+\cos \theta_{0} \tilde{\theta}_{1}\right)-\tilde{T}_{2}\left(\sin \theta_{0}+\cos \theta_{0} \tilde{\theta}_{2}\right)\right) .
\end{aligned}
$$

Solving these equations at $x \neq 0$ we obtain $\tilde{\theta}_{1(2)}=\alpha_{1(2)} e^{-x / \lambda}+\beta_{0} x$ and $\theta_{s}=\beta_{s} x$ where $\tilde{K}_{1} \alpha_{1}=-\tilde{K}_{2} \alpha_{2}$ and $\frac{1}{\lambda^{2}}=|\tilde{J}| \frac{\left(\tilde{K}_{1}+\tilde{K}_{2}\right)}{\tilde{K}_{1} \tilde{K}_{2}}$.

Matching the boundary condition at $x=0$ we also obtain

$$
\begin{aligned}
J_{1}=\tilde{K}_{1}\left(\beta_{0}-\frac{\alpha_{1}}{\lambda}\right) & =\tilde{T}_{1}\left(\sin \theta_{0}+\cos \theta_{0} \alpha_{1}\right), \\
J_{2}=\tilde{K}_{2}\left(\beta_{0}-\frac{\alpha_{2}}{\lambda}\right) & =-\tilde{T}_{2}\left(\sin \theta_{0}+\cos \theta_{0} \alpha_{2}\right), \\
\tilde{K}_{s} \beta_{s} & =J_{1}+J_{2} .
\end{aligned}
$$

The first two equations give the tunnelling currents flowing from band one (two) to the singleband superconductor, respectively. The third equation expresses total current conversation across the Josephson junction.

We shall concentrate on the ground state solution where the Josephson junction does not introduce any bulk energy cost. In this case there is no net current flowing through the system and $\beta_{0}=\beta_{s}=0$. Solving Eq. (6) we find that only one solution $\cos \theta_{0}=\operatorname{sgn}\left(\tilde{T}_{1}-\tilde{T}_{2}\right)$ exists at $\left|\tilde{T}_{1}-\tilde{T}_{2}\right|>\sigma_{1}$, where $\sigma_{1}=\frac{\lambda \tilde{T}_{1} \tilde{T}_{2}\left(\tilde{K}_{1}+\tilde{K}_{2}\right)}{\tilde{K}_{2} \tilde{K}_{1}}$ whereas two possible solutions $\cos \theta_{0}=$ $\left(\operatorname{sgn}\left(\tilde{T}_{1}-\tilde{T}_{2}\right), \frac{\left(\tilde{T}_{1}-\tilde{T}_{2}\right)}{\sigma_{1}}\right)$ exist at $\left|\tilde{T}_{1}-\tilde{T}_{2}\right|<\sigma_{1}$. The true solution at $\left|\tilde{T}_{1}-\tilde{T}_{2}\right|<\sigma_{1}$ is the one with lower energy. Comparing the energies $F\left(\theta_{0}, \tilde{\theta}\right)$ of the two states we find

$$
\begin{array}{rlr}
\cos \theta_{0} & =\operatorname{sgn}\left(\tilde{T}_{1}-\tilde{T}_{2}\right) \quad\left(\left|\tilde{T}_{1}-\tilde{T}_{2}\right|>\sigma\right) \\
& =\frac{\left(\tilde{T}_{1}-\tilde{T}_{2}\right)}{\sigma_{1}} \quad\left(\left|\tilde{T}_{1}-\tilde{T}_{2}\right|<\sigma\right)
\end{array}
$$

where $\sigma=\min \left(\sigma_{1}, \sigma_{2}\right), \sigma_{2}=\frac{\lambda\left(\tilde{K}_{2} \tilde{T}_{1}+\tilde{K}_{1} \tilde{T}_{2}\right)^{2}}{\left(\tilde{K}_{1}+\tilde{K}_{2}\right) \tilde{K}_{2} \tilde{K}_{1}}$.

In the first case $\theta_{0}=0$ or $\pi$, which corresponds to the first type of solution in the classical spin problem. The solution respects time-reversal symmetry and we shall call it TRI state in the following.

There are two degenerate solutions in the second case corresponding to $\theta_{0} \gtrless 0$ which are time-reversal pairs $\left(\psi_{1(2)} \rightleftharpoons \psi_{1(2)}^{*}\right)$. The solution breaks time reversal symmetry and we shall call it the $T R B$ state. The corresponding $\alpha_{1(2)}$ is equal to zero in the $T R I$ state, and 
is of order $\sin \theta_{0} \tilde{T}_{1(2)} \lambda / \tilde{K}_{1(2)}$ in the $T R B$ state, which is much less than $\theta_{0}$ in the limit of weak-Josephson coupling $\tilde{T}_{1(2)} \lambda / \tilde{K}_{1(2)}<<1$, justifying our approximate treatment of free energy.

It is interesting to note that although the net Josephson current passing through the tunnelling barrier is zero in the ground state, the currents $J_{1}$ and $J_{2}=-J_{1}$, which represent current passing from band 1(2) of the two-band superconductor to the single-band superconductor, are nonzero in the $T R B$ state. Correspondingly there is also a nonzero current $J_{12} \sim \tilde{J} \sin \left(\theta_{1}-\theta_{2}\right)$ passing from band one to band two in the TRB state.

Thus the TRB state is characterized by a novel current "loop" through the Josephson function. A current flows from band one/two of the two-band superconductor to the singleband superconductor through the Josephson junction, and flows back to band two/one of the two-band superconductor. The current flow from band two/one to band one/two inside the two-band superconductor to complete the current loop. The current loop we see here is not a current loop circulating in real space, but a current loop in $\vec{k}$-space, if we envision the two bands as occupying different parts of the $\vec{k}$-space.

It is also straightforward to see from Eq. (6) that the parameter space where the TRB state exists is enlarged when there is a net current flowing across the Josephson junction $\left(\beta_{0}, \beta_{s} \neq 0\right)$. This is not surprising since a finite current through the system breaks timereversal symmetry. The current also removes the degeneracy of the two $T R B$ solutions with $\theta_{0} \lessgtr 0$.

The presence of non-trivial phase structure leads to non-trivial electron surface states on the surface of the Josephson junction. To study these surface states we consider the ground state and analyze the corresponding Bogoliubov-de Gennes (BdG) equation,

$$
\begin{aligned}
& \epsilon_{n}^{(i)} u_{n}^{(i)}(\vec{x})=\hat{H}_{o}^{(i)} u_{n}^{(i)}(\vec{x})+\Delta^{(i)}(\vec{x}) v_{n}^{(i)}(\vec{x}) \\
& \epsilon_{n}^{(i)} v_{n}^{(i)}(\vec{x})=-\hat{H}_{o}^{(i)} v_{n}^{(i)}(\vec{x})+\Delta^{(i) *}(\vec{x}) u_{n}^{(i)}(\vec{x})
\end{aligned}
$$

where $i=1,2$ are the band indices, $\hat{H}_{o}^{(i)}$ gives the single-particle band structure for band $i$ and $\Delta^{(i)}(\vec{x})$ is the corresponding superconducting order parameter. We have assumed that the two bands are independent of each other and are coupled implicitly only through the superconductor order parameter in writing down Eq.(8) . In particular, $\Delta^{(1)} \sim+(-) \Delta^{(2)}$ in the bulk superconductor if $J>(<) 0$. The Josephson function can be modeled by superconducting order parameters of the form $\Delta^{(i)}(\vec{x}) \sim \Delta_{0}^{(i)} e^{i \theta_{i}(x)}$ at $x>0$ and $\Delta(x)=\Delta_{s}$ at $x<0$, 
where $\Delta_{0}^{(i)}$ and $\Delta_{s}$ are real and positive. We shall show in the following that non-trivial electronic surface state exists in the Josephson junction, with structures depending strongly on the phases of the superconducting order parameters.

The BdG equations for electronic states close to the Fermi surface can be solved in the WKBJ approximation 13 where we write $u(v)_{n}^{(i)}(\vec{x}) \sim e^{i \vec{k}_{F} \cdot \vec{x}} \tilde{u}(\tilde{v})_{n}^{(i)}(\vec{x})$, where $\tilde{u}(\tilde{v})_{n}^{(i)}(\vec{x})$ are slowly varying functions of $\vec{x}$ (on the scale of $k_{F}^{-1}$ ) satisfying the Andreev equations,

$$
\begin{aligned}
& \epsilon_{n}^{(i)} \tilde{u}_{n}^{(i)}(\vec{x})=-i\left(\vec{v}_{F}^{(i)} \cdot \nabla\right) \tilde{u}_{n}^{(i)}(\vec{x})+\Delta^{(i)}(\vec{x}) \tilde{v}_{n}^{(i)}(\vec{x}) \\
& \epsilon_{n}^{(i)} \tilde{v}_{n}^{(i)}(\vec{x})=i\left(\vec{v}_{F}^{(i)} \cdot \nabla\right) \tilde{v}_{n}^{(i)}(\vec{x})+\Delta^{(i) *}(\vec{x}) \tilde{u}_{n}^{(i)}(\vec{x}),
\end{aligned}
$$

where $\vec{v}_{F}^{(i)}$ is the Fermi velocity of the band $i$ electrons with momentum $\vec{k}_{F}^{(i)}$. We shall consider the weak-Josephson coupling limit $\left(\tilde{\theta}_{1(2)}<<\theta_{0}\right)$ in the following, so that $\theta_{1}(x) \sim \theta_{0}$ and $\theta_{2}(x) \sim \theta_{0}-\pi$. The bound states are given by solutions of form $\tilde{u}(\tilde{v})_{n}(\vec{x})=\tilde{u}(\tilde{v})_{0} e^{-\gamma_{+} x}$ for $x>0$ and $\tilde{u}(\tilde{v})_{n}(\vec{x})=\tilde{u}(\tilde{v})_{0} e^{\gamma_{-} x}$ for $x<0$. Substituting these into Eq. (9), we obtain the self-consistent equations

$$
\begin{aligned}
\epsilon_{0}^{(i) 2}= & \Delta_{0}^{(i) 2}\left(1-x^{(i) 2}\right)=\Delta_{s}^{2}\left(1-y^{(i) 2}\right) \\
\frac{\epsilon_{0}^{(i)} / \Delta_{0}^{(i)}-i x^{(i)}}{\epsilon_{0}^{(i)} / \Delta_{s}+i y^{(i)}} & =e^{i \theta_{i}}
\end{aligned}
$$

where $x^{(i)}=\vec{v}_{F}^{(i)} \cdot \hat{x}\left(\gamma_{+}^{(i)} / \Delta_{0}^{(i)}\right)$ and $y^{(i)}=\vec{v}_{F}^{(i)} \cdot \hat{x}\left(\gamma_{-}^{(i)} / \Delta_{s}\right)$. Notice that $x^{(i)}$ and $y^{(i)}$ must have the same sign in this representation and changing sign of $x(y)^{(i)}$ corresponds to changing $\vec{v}_{F} \rightarrow-\vec{v}_{F}$.

It is easy to see that if $x^{(i)}, y^{(i)}$ is a solution of Eq. (10) with energy $\epsilon_{0}^{(i)}$, then $-x^{(i)},-y^{(i)}$ is a solution with energy $-\epsilon_{0}^{(i)} .-x^{(i)},-y^{(i)}$ is also a solution with energy $\epsilon_{0}^{(i)}$ with $\theta_{i} \rightarrow-\theta_{i}$. Therefore it is sufficient to consider the range $\pi>\theta_{i}>0$. Solving Eq (10) we find that solutions where $x^{(i)}$ and $y^{(i)}$ have the same sign exists only when $\cos \theta_{i}<\min \left(\Delta_{0}^{(i)} / \Delta_{s}, \Delta_{s} / \Delta_{0}^{(i)}\right)$, with

$$
\begin{aligned}
x^{(i)} & =\frac{\Delta_{s} \cos \theta_{i}-\Delta_{0}^{(i)}}{D^{(i)}} \operatorname{sgn}\left(\epsilon_{0}^{(i)}\right), \\
y^{(i)} & =\frac{\Delta_{0}^{(i)} \cos \theta_{i}-\Delta_{s}}{D^{(i)}} \operatorname{sgn}\left(\epsilon_{0}^{(i)}\right), \\
\epsilon_{0}^{(i)} & = \pm \frac{\Delta_{0}^{(i)} \Delta_{s} \sin \theta_{i}}{D^{(i)}} .
\end{aligned}
$$

where $D^{(i)}=\sqrt{\Delta_{s}^{2}+\Delta_{0}^{(i) 2}-2 \cos \theta_{i} \Delta_{s} \Delta_{0}^{(i)}}$. Notice that there exists one solution for each 
value of Fermi momentum $\vec{k}_{F}^{(i)}=\left(k_{F x}^{(i)}, k_{F y}^{(i)}, k_{F z}^{(i)}\right)$. Thus a finite density of states exist at the Josephson junction in general.

We now analyze the solutions as a function of $\theta_{i}$. First we note that bound state solutions do not exist when $\theta_{i}=0$, i.e. when $J>0$ and the system is not frustrated. For $J<0$ and $\theta_{i}=\pi$, we find that bound state solutions with $\epsilon_{0}^{(i)}=0$ exist at both $x(y)^{(i)} \lessgtr 0$ channels. In the corresponding Josephson junction problem, bound state solutions with zero energy exist in the $T R I$ state in the band which is out-of-phase with the single-band superconductor, but there is no bound state solution for the band which is in-phase with the single-band superconductor. Since time-reversal symmetry is preserved in the TRI state, the $x(y)^{(i)} \lessgtr 0$ states have the same bound state energy $\epsilon_{0}^{(i)}=0$.

The bound state structure is much richer in the TRB state which breaks time-reversal symmetry. In this case, the structure of the bound states depend on the value of $\theta_{0}$ (Recall $\theta_{1} \sim \theta_{0}$ and $\left.\theta_{2} \sim \theta_{0}-\pi\right)$. First we consider $\theta_{0}<\pi / 2$. For small $\theta_{0}$ such that $\cos \theta_{0}>$ $\min \left(\Delta_{0}^{(1)} / \Delta_{s}, \Delta_{s} / \Delta_{0}^{(1)}\right)$, bound state solutions exist only in band two. The bound states exist in pairs with energies $\pm \epsilon_{0}^{(2)} \neq 0$, corresponding to time-reversal pairs $x(y)^{(2)} \lessgtr 0$. For larger $\theta_{0}$ such that $\cos \theta_{0}<\min \left(\Delta_{0}^{(1)} / \Delta_{s}, \Delta_{s} / \Delta_{0}^{(1)}\right)$, bound state solutions exist in both bands in time-reversal pairs with energies $\pm \epsilon_{0}^{(1,2)} \neq 0$. Similar results occur for $\theta_{0}>\pi / 2$ where bound states exist only in band one if $-\cos \theta_{0}>\min \left(\Delta_{0}^{(2)} / \Delta_{s}, \Delta_{s} / \Delta_{0}^{(2)}\right)$ and exist in both bands if $-\cos \theta_{0}<\min \left(\Delta_{0}^{(2)} / \Delta_{s}, \Delta_{s} / \Delta_{0}^{(2)}\right)$.

Now the experimental observation of this bound state is discussed. First, the $d I / d V$ curve for the Josephson tunneling should show the peak at the bound state energy within the gap. A zero-bias peak would exists in the $T R I$ state, and is split into two peaks centered at finite energies in the TRB state. The local probe such as the STS can also be used to detect these bound states. The detection of the persistent Josephson current loop in $\vec{k}$-space in the $T R B$ state will be a challenge. The standard prove for the time-reversal symmetry breaking is the Kerr rotation. With the spin-orbit interaction, the finite spin density is expected at the Josephson junction, but the details of the analysis depends on band structure which we have not undertaken in this paper.

We emphasize that the effects discussed in this paper are general effects associated with frustrated phase structures in superconductor Josephson junctions, and is not restricted to $s$-wave superconductor, or Josephson junction involving single-band and two-band superconductors. This idea of the frustration can be generalized to single multi-band superconductors 
with three or more bands coupled via internal Josephson effect. In this case, we can construct the effective XY-spin model with positive or negative exchange interactions between pairs of the order parameters, and when there exists relative angle(s) different from 0 or $\pi$, time-reversal symmetry is broken spontaneously. This mechanism is likely to be active in the superconductors with rather complex band structure such as the heavy fermion systems, where many sheets of the Fermi surface contribute to the pairing.

To summarize, we have studied the Josephson junction between the two-gap superconductor and the conventional s-wave superconductor. When the relative sign of the two gaps are negative, the Josephson coupling introduces the frustration, which can lead to the time-reversal symmetry breaking near the junction. This results in the bound state within the gaps, which an be detected by the $d I / d V$ characteristics or STS, which can offers an experimental test of the relative phase of the two gaps.

The authors are grateful to Patrick A Lee for fruitful discussions. This work was supported in part by Grant-in-Aids (Grant No. 15104006, No. 16076205, and No. 17105002) and NAREGI Nanoscience Project from the Ministry of Education, Culture, Sports, Science, and Technology. TKN also acknowledge support by HKUGC through grant CA05/06.Sc04.

1 H. Suhl, B.T. Matthias, and L.R. Walker, Phys. Rev. Lett. 12, 552 (1959).

2 J. Nagamatsu et al., Nature 410, 63 (2001)

3 F. Bouquet et al. Phys. Rev. Lett. 87, 047001 (2001)

4 P. Szabo et al., Phys. Rev. Lett. 87, 137005 (2001)

5 S. Souma et al., Nature b 473, 65 (2003)

6 H. Choi et al., Nature 418, 758 (2002)

7 Y. Kamihara et al., J. Am. Chem. Soc. 130, 3296 (2008).

8 D. J. Singh and M. H. Du, arXiv:0803.0429.

9 Fe Wang et al., arXiv:0807.0498 and references therein.

10 H. Ding et al., Europhys. Lett. 83, 47001 (2008).

11 A. D. Christianson et al., arXiv:0807.3932,

12 J. Chang et al., Phys. Rev. B 75, 24503 (2007).

13 C.-R. Hu, Phys. Rev. Lett. 72, 1526 (1994). 